\title{
miR-486-5p inhibits cell proliferation and invasion through repressing GAB2 in non-small cell lung cancer
}

\author{
SUNFENG YU* ${ }^{*}$ SHUANG GENG* ${ }^{*}$ and YI HU \\ Department of Respiratory Medicine, The Central Hospital of Wuhan, Tongji Medical College, \\ Huazhong University of Science and Technology, Wuhan, Hubei 430014, P.R. China
}

Received February 2, 2018; Accepted June 19, 2018

DOI: $10.3892 / 01.2018 .9053$

\begin{abstract}
Previous studies have reported that cell metastasis is the main reason for the high mortality of non-small cell lung cancer (NSCLC). Many miRNAs have been identified to be involved in the development of NSCLC. In this study, we explored the effect of miR-486-5p and GAB2 on cell proliferation and invasion in NSCLC. First, miR-486-5p and GAB2 expression levels were detected in NSCLC through quantitative RT-qPCR, and downregulation of miR-486-5p and upregulation of GAB2 were both identified in NSCLC. Then MTT and Transwell analysis were performed to confirm the functions of miR-486-5p and GAB2 for cell proliferation and invasion in NSCLC. Moreover, miR-486-5p overexpression was found to inhibit proliferation and invasion by suppressing GAB2 in NSCLC cells. Besides, miR-486-5p overexpression lessened GAB2 expression level in NSCLC, while miR-486-5p knockout enhanced GAB2 expression level. Additionally, miR-486-5p was identified to directly target GAB2 through dual luciferase reporter assay. The silence of GAB2 was found to inhibit proliferation and invasion of NSCLC cells. Collectively, miR-486-5p contributed to inhibiting proliferation and invasion of NSCLC cells through regulating GAB2, and miR-486-5p/GAB2 axis may provide a breakthrough for diagnosing NSCLC.
\end{abstract}

\section{Introduction}

The growth rate and metastasis of non-small cell lung cancer (NSCLC) have been reported to be slower and later than that of small cell carcinoma (1). Moreover, NSCLC accounts

Correspondence to: $\mathrm{Dr} \mathrm{Yi} \mathrm{Hu}$, Department of Respiratory Medicine, The Central Hospital of Wuhan, Tongji Medical College, Huazhong University of Science and Technology, 26 Shengli Street, Jiang'an, Wuhan, Hubei 430014, P.R. China

E-mail: d4000084748qe@163.com

${ }^{*}$ Contributed equally

Key words: miR-486-5p, non-small cell lung cancer, invasion, proliferation, GAB2 for $80-85 \%$ of the total lung cancer, and now chemotherapy is the main treatment for NSCLC (2). Moreover, low overall 5-year survival and high recurrence rate of NSCLC patients (3) make it urgent to develop novel treatments for NSCLC. Therefore, future direction is to further analyze potential therapeutic targets in the process of NSCLC occurrence.

Recently, microRNA has been paid increasing attention in cancer research in regulation of many biological processes including growth, proliferation, migration, invasion, and apoptosis (4-6). Especially in NSCLC, some downregulated miRNAs such as miR-134, miR-204, miR-34c, and miR-200b were reported to repress the development of NSCLC (7-10). In addition, many upregulated miRNAs such as miR-21, miR-205, miR-211, and miR-484 were reported to promote cell growth, cell proliferation, cell cycle and invasion (11-14). These previous studies elucidated that the alteration of miRNA expression could affect tumorigenesis of NSCLC.

Among these miRNAs, miR-486-5p has been identified in various human cancers and diseases. It has been reported that miR-486-5p was downregulated in hepatocellular carcinoma (15), breast cancer (16), duchenne muscular dystrophy (17) and chronic kidney disease (18). However, studies are rare on miR-486-5p in NSCLC. Based on the above research, we speculated that miR-486-5p expression level might be declined in regulation of the progression of NSCLC. Additionally, targeted genes such as NOB1 (19), RUNX3 (20) and FOXM1 (21) have been found to influence the tumorigenesis of NSCLC. Nonetheless, the role of GAB2 in NSCLC is rarely reported.

In the present study, we explored the effect of miR-486-5p on the NSCLC development. As predicted, miR-486-5p expression was decreased in NSCLC. Moreover, miR-486-5p repressed cell proliferation and invasion in NSCLC through suppressing GAB2. These findings provide a new way for treatment of NSCLC.

\section{Materials and methods}

Clinical tissues. Forty-six NSCLC and adjacent tissues were obtained from The Central Hospital of Wuhan, Tongji Medical College, Huazhong University of Science and Technology (Wuhan, China) after receiving written informed consent. None of the patients received treatment prior to the operation. Then the tissues were frozen in liquid nitrogen and stored at 
$-80^{\circ} \mathrm{C}$ in a refrigerator for further experiment. This study was approved by the Central Hospital of Wuhan, Tongji Medical College, Huazhong University of Science and Technology institutional Ethics Committee.

Cell cultures and cell transfection. The human NSCLC cell lines A549, SPC-A1 and BEAS-2B (control) were used for this study. All the cell lines were obtained from the Shanghai Cell Bank, China Academy of Sciences (Shanghai, China). Then these cell lines were seeded in DMEM medium (Cellgro; Corning Incorporated, Corning, NY, USA) with $10 \%$ fetal bovine serum (FBS) and cultured at $37^{\circ} \mathrm{C}$ with $5 \% \mathrm{CO}_{2}$.

The miR-486 mimic/mimic control (miR10004762-1-5/ miR01201-1-5), the miR-486 inhibitor/inhibitor control (miR20004762-1-5/miR02201-1-5) and the GAB2 siRNA (si-GAB2, 5'-AAA CGC UGG UUU AUA CUG CGG-3'), purchased from RiboBio Co., Ltd. (Guangzhou, China), were transferred into NSCLC cells by Lipofectamine 2000 (Thermo Fisher Scientific, Inc., Waltham, MA, USA) based on the manufactures' instructions.

Quantitative RT-qPCR. TRIzol reagent (Invitrogen; Thermo Fisher Scientific, Inc.) was applied for extracting total RNA containing miRNA to quantitate miR-486-5p expression in NSCLC. To obtain the cDNA templates, $1 \mu \mathrm{g}$ total RNA of each sample was used for reverse transcription using a miScript Reverse Transcription kit (Qiagen $\mathrm{GmbH}$, Hilden, Germany). This reaction was performed at $37^{\circ} \mathrm{C}$ for $60 \mathrm{~min}$, then $95^{\circ} \mathrm{C}$ for $5 \mathrm{~min}$. Quantitative RT-qPCR was carried out through the SYBR-Green PCR kit (Takara Bio, Inc., Otsu, Japan) on ABI 7500 Fast Real-Time PCR system (ABI; Thermo Fisher Scientifc, Inc.). The cycling conditions for RT-qPCR were as follows: $5 \mathrm{~min}$ at $95^{\circ} \mathrm{C}$, followed by 40 cycles of $95^{\circ} \mathrm{C}$ for $30 \mathrm{sec}$ and $60^{\circ} \mathrm{C}$ for $45 \mathrm{sec}$. U6 and GAPDH were used as control for miR-486-5p and GAB2. The primers were: GAPDH forward, 5'-TGTTCGTCATGGGTGTGAAC-3' and reverse, 5'-ATGGCATGGACTGTGGTCAT-3'; GAB2 forward, 5'-CGAAGAGAACTATGTCCCTATGC-3'; reverse, 5'-AGGGGCAGGACTGTTCGT-3' miR-486-5p forward, 5'-ACACTCCAGCTGGGTCCTGTACTGAGCTGCCC-3' and reverse, 5'-CTCAACTGGTGTCGTGGAGTCGGCAAT TCAGTTGAGCCCCGAG-3'; U6 forward, 5'-CTCGCT TCGGCAGCACA-3' and reverse, 5'-AACGCTTCACGA ATTTGCGT-3'. The expression was calculated using the $2^{-\Delta \Delta c q}$ method (22).

Luciferase activity assay. The wild or mutant type of 3'-UTR of GAB2 was inserted into the pGL3 promoter vector (Invitrogen; Thermo Fisher Scientific, Inc.) for luciferase reporter experiments. GAB2 vector (pCDNA3.1-GAB2) was purchased from RiboBio Co., Ltd. Then, we transfected GAB2 vector and miR-486-5p mimic into A549 cells. Subsequently, the dual luciferase reporter assay (Promega Corporation, Madison, WI, USA) was applied to perform luciferase assays.

MTT assay. The MTT (3-(4,5-dimethyl-2-thiazolyl)-2,5-diphenyl-2H-tetrazolium bromide) assay was applied to measure cell proliferation. The cells $\left(4 \times 10^{3}\right.$ cells/well) were added onto 96-well plates in medium with $10 \%$ FBS. The cells with miR-486-5p mimic or inhibitor were incubated for $0-72 \mathrm{~h}$.
After incubation, the cells added with MTT (Sigma-Aldrich; Merck KGaA, Darmstadt, Germany) and were cultured at $37^{\circ} \mathrm{C}$ for $4 \mathrm{~h}$. The absorbance at $490 \mathrm{~nm}(\mathrm{OD}=490 \mathrm{~nm})$ was detected with a spectrophotometer (Model 680 microplate reader; Bio-Rad Laboratories, Inc., Hercules, CA, USA).

Cell invasion assay. The cells were plated into the upper chambers ( $8 \mu \mathrm{m}$ pore size; Corning Incorporated) and medium with $10 \%$ FBS was put into the lower chamber. Then these cells $\left(5 \times 10^{3}\right)$ were cultured for $24 \mathrm{~h}$ at $37^{\circ} \mathrm{C}$ in atmosphere with $5 \%$ $\mathrm{CO}_{2}$. Then the invasive cells in the lower chamber were fixed with $70 \%$ ethanol and stained using crystal violet. Finally, light microscope (DM IRB; Leica Microsystems GmbH, Wetzlar, Germany) was used to measure the cell number.

Western blot analysis. The protein samples were obtained using RIPA lysis buffer. 10\% SDS-PAGE was employed to separate proteins which were incubated with $5 \%$ non-fat milk in polyvinylidene difluoride membranes (EMD Millipore, Billerica, MA, USA) at room temperature. Next, we incubated the membranes overnight at $4^{\circ} \mathrm{C}$ with rabbit monoclonal anti-GAB2 (1:1,000; catalog no. ab203478; Abcam, Shanghai, China), rabbit polyclonal anti-GAPDH $(1: 1,000$; catalog no. ab70699; Abcam) and subsequently incubated with goat anti-rabbit IgG-H\&L secondary antibody $(1: 1,000$; catalog no. ab150077; Abcam). Then, protein expression levels were measured by an Enhanced Chemiluminescence Immunoblot Detection system (Pierce; Thermo Fisher Scientific, Inc.) and analyzed using Quantity One software (version 4.62; Bio-Rad Laboratories, Inc., Hercules, CA, USA).

Statistical analysis. The obtained data are shown as the mean \pm standard deviation. The difference between the groups was calculated through Chi-square test or one-way ANOVA with Tukey's as a post hoc test. Statistical analysis was analyzed with GraphPad Prism 6.0 (GraphPad Software, Inc., La Jolla, CA, USA) and SPSS 19.0 (IBM Corp., Armonk, NY, USA). $\mathrm{P}<0.05$ was considered to indicate a statistically significant difference.

\section{Results}

Expression of miR-486-5p and GAB2 mRNA was detected in NSCLC. First, we detected miR-486-5p expressions in NSCLC via quantitative RT-qPCR. The results suggested that miR-486-5p expression was reduced in NSCLC tissues compared with normal tissues (Fig. 1A). Moreover, the downregulation of miR-486-5p was also identified in SPC-A1 and A549 cell lines (Fig. 1B). Subsequently, the expression of GAB2 was analyzed in NSCLC as well. Interestingly, GAB2 mRNA expression was increased in NSCLC tissues and cell lines (Fig. 1C and D) which was contrary to miR-486-5p expression.

miR-486-5p suppresses cell proliferation and invasion in $N S C L C$. The function of miR-486-5p in NSCLC was verified by performing MTT and Transwell assay in A549 cells containing miR-486-5p mimic or inhibitor. Primarily, the miR-486-5p expression level was detected in cells with miR-486-5p mimic or inhibitor as shown in Fig. 2A and B. Importantly, MTT 

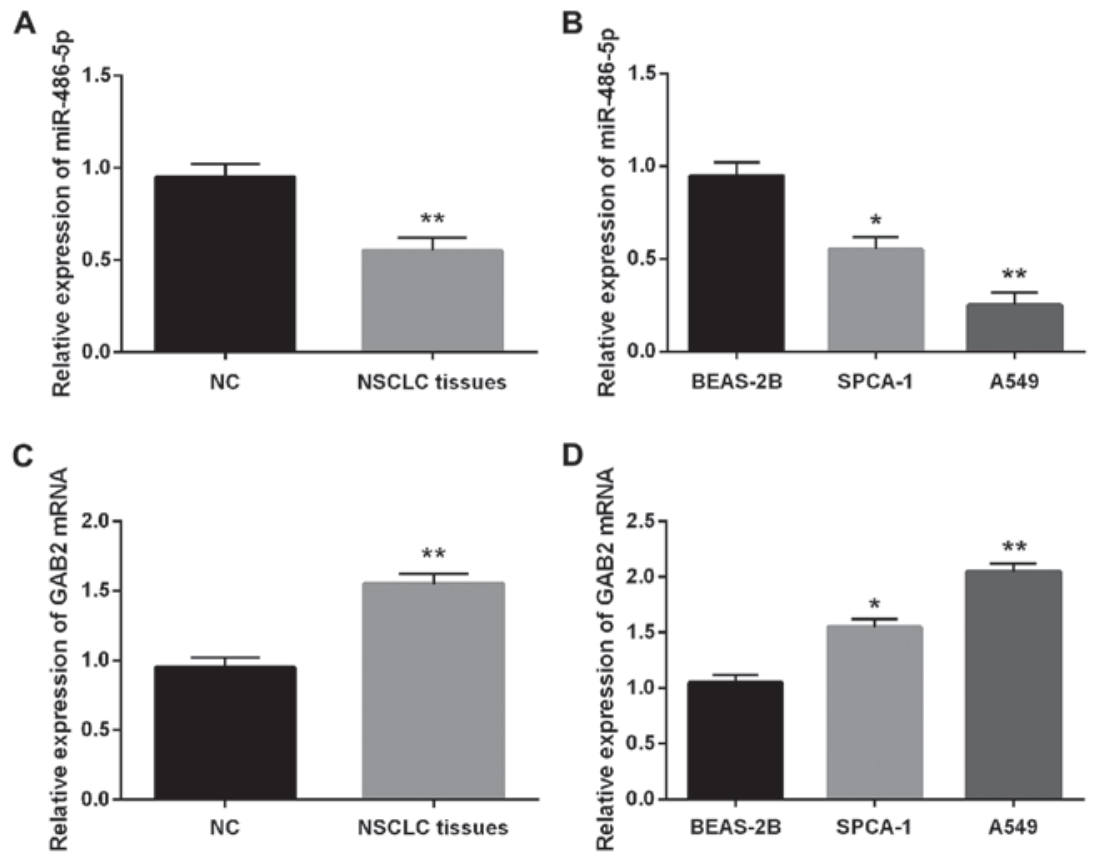

Figure 1. Expression of miR-486-5p and GAB2 mRNA was detected in NSCLC. (A) The miR-486-5p expression in NSCLC tissues and normal tissues were detected via RT-qPCR. (B) The miR-486-5p level in SPC-A1, A549 and BEAS-2B cells (control). (C) GAB2 mRNA expression in NSCLC tissues were detected via RT-qPCR. (D) GAB2 mRNA expression in SPC-A1, A549 and BEAS-2B cells (control). ${ }^{*} \mathrm{P}<0.05,{ }^{* * *} \mathrm{P}<0.01$. NSCLC, non-small cell lung cancer.

A

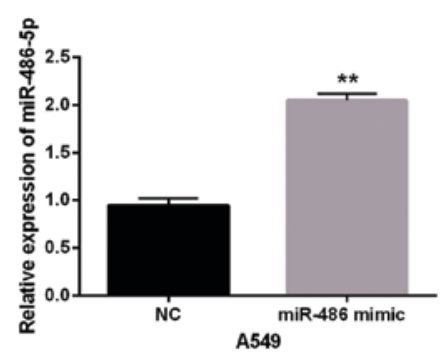

C

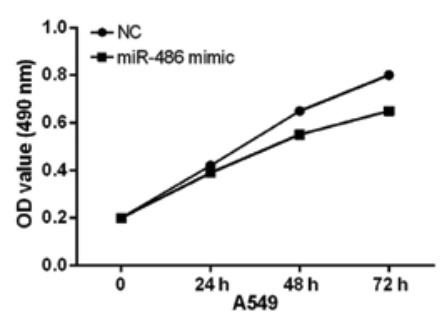

E

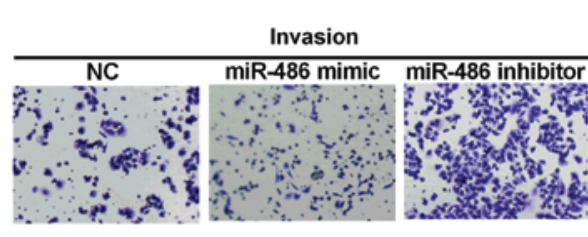

B

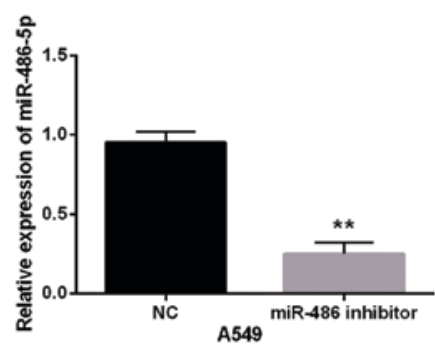

D
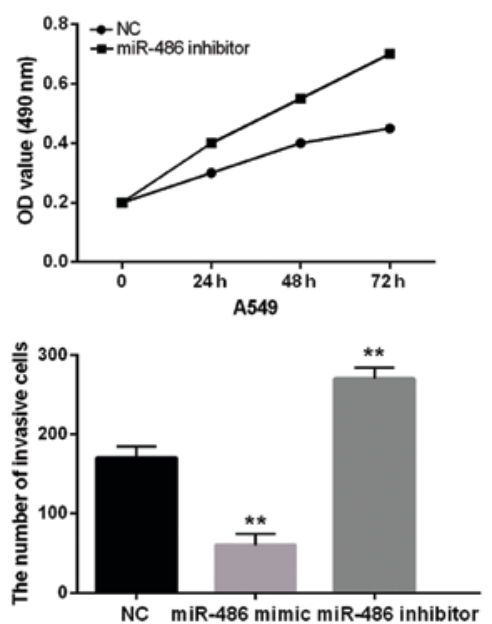

A549

Figure 2. The function of miR-486-5p for cell proliferation and invasion in NSCLC. (A and B) The expression of miR-486-5p was examined in A549 cells contained miR-486-5p mimics or inhibitor via RT-qPCR. (C and D) Cell proliferation was measured in cells that contained miR-486-5p mimics or inhibitor via MTT. (E) The cell invasion was measured in cells that contained miR-486-5p mimics or inhibitor via Transwell analysis. ${ }^{* *} \mathrm{P}<0.01$. NSCLC, non-small cell lung cancer.

assay showed that miR-486-5p overexpression suppressed cell proliferation, while miR-486-5p downregulation exhibited the opposite result in NSCLC (Fig. 2C and D). Transwell assay suggested that the cell invasion was significantly repressed 


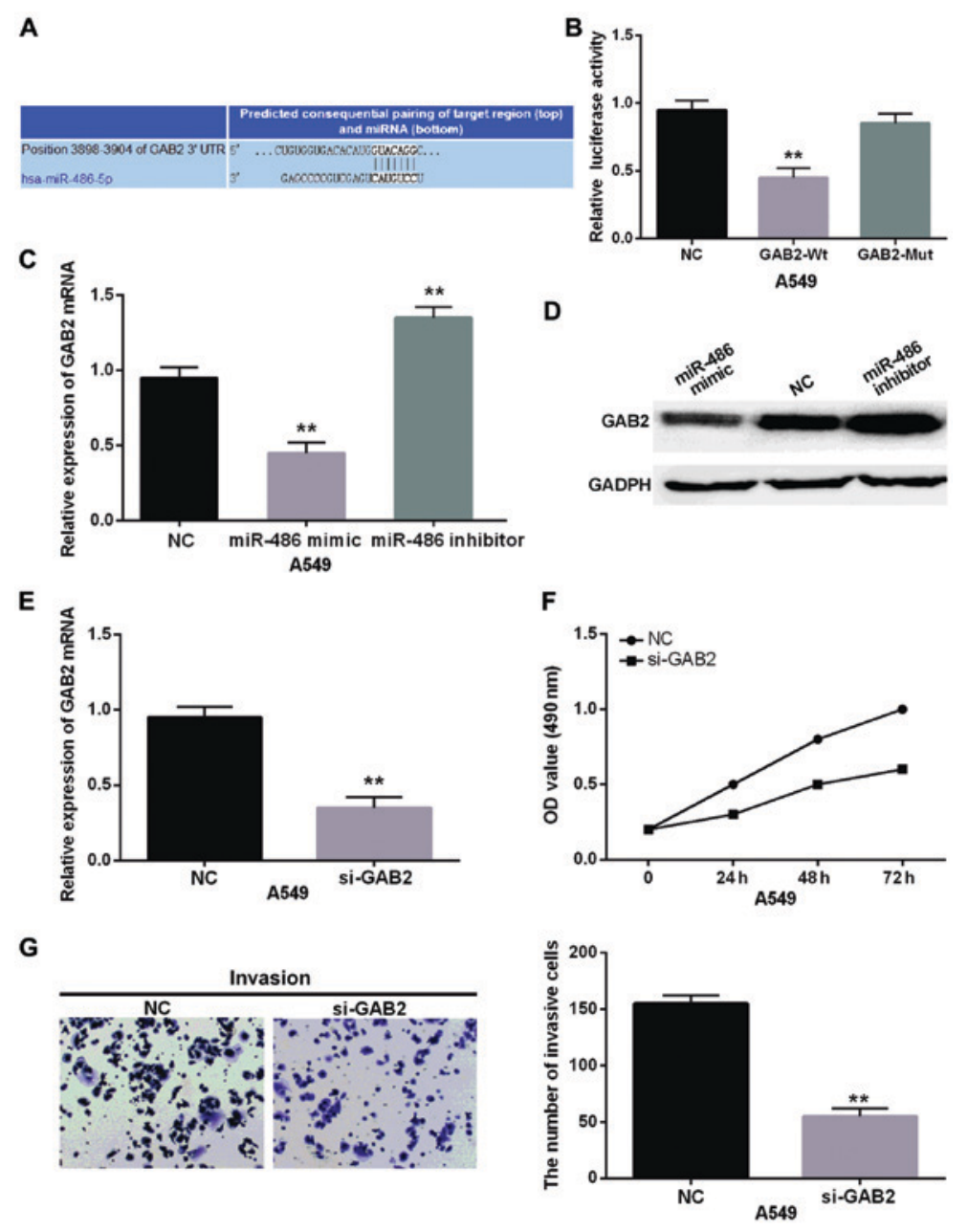

Figure 3. GAB2 is a direct target gene of miR-486-5p and has effect on NSCLC. (A) The binding sites of miR-486-5p on the 3'UTR of GAB2. (B) Luciferase reporter assay. (C and D) GAB2 mRNA and protein levels were analyzed in cells containing miR-486-5p mimic or inhibitor. (E) The mRNA expression of GAB2 was measured in cells containing GAB2 siRNA. (F) The cell proliferation was identified in cells containing si-GAB2. (G) Cell invasion analysis in cells with si-GAB2 was also detected in NSCLC cells. ${ }^{* *} \mathrm{P}<0.01$. NSCLC, non-small cell lung cancer.

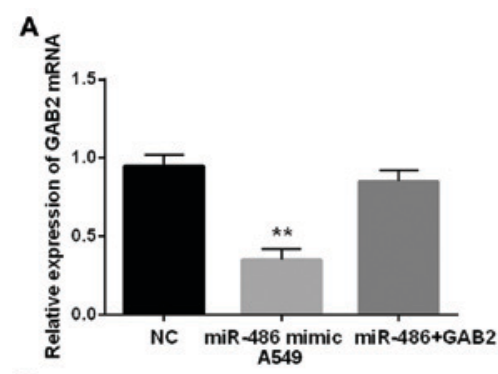

B

D

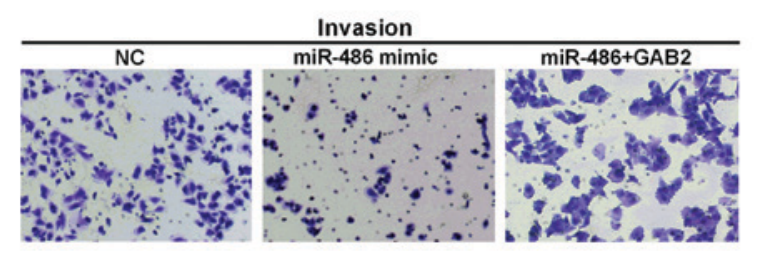

C
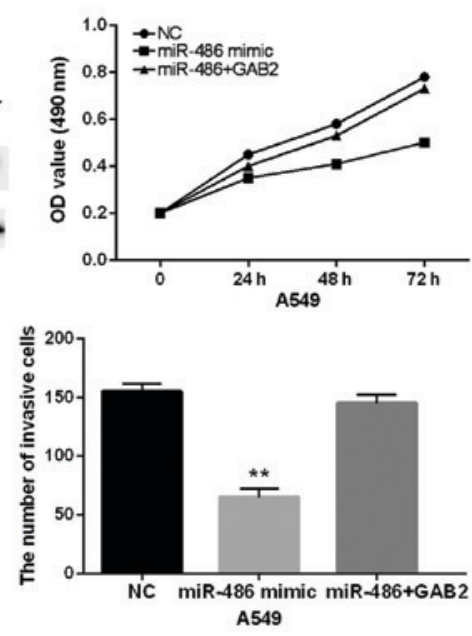

Figure 4. miR-486-5p regulates cell proliferation and invasion through suppressing GAB2 in NSCLC. (A and B) GAB2 mRNA and protein levels were measured in cells containing miR-486-5p and GAB2 vector. (C) The cell proliferation was detected in cells containing miR-486-5p and GAB2 vector. (D) The cell invasion was examined in cells containing miR-486-5p and GAB2 vector. ${ }^{* *} \mathrm{P}<0.01$. NSCLC, non-small cell lung cancer.

by miR-486-5p overexpression. However, it was promoted by miR-486-5p inhibitor in NSCLC cells (Fig. 2E). These findings indicated that cell proliferation and invasion were inhibited in NSCLC by miR-486-5p overexpression. 
GAB2 is a direct target gene of $m i R-486-5 p$ in NSCLC. The target genes of miR-486-5p were searched through the database of TargetScan Human (http://www.targetscan. org/vert_71/), which indicated that it binds with the 3'-UTR of GAB2 (Fig. 3A). Moreover, we confirmed that miR-486-5p directly targeted GAB2 and had a binding site with the wildtype of GAB2 by dual luciferase reporter assay (Fig. 3B). Additionally, mRNA and protein expression of GAB2 were reduced by miR-486-5p mimics whereas increased by miR-486-5p inhibitor (Fig. 3C and D). Furthermore, si-GAB2 was transfected into A549 cells to further explore its role in NSCLC (Fig. 3E). Additionally, we found that si-GAB2 had the similar effect as the upregulation of miR486-5p which suppressed cell proliferation and invasion in NSCLC (Fig. 3F and G). Therefore, it was inferred that miR-486-5p directly targeted GAB2 and knockout of GAB2 inhibited NSCLC cell proliferation and invasion.

miR-486-5p regulates cell proliferation and invasion through suppressing GAB2 in NSCLC. Finally, we transfected GAB2 vector and miR-486-5p mimics into NSCLC cells to verify their relationship, and we found that there was almost no change in expression of mRNA and protein of GAB2 in cells with miR-486-5p mimic and GAB2 vector in comparison with the control (Fig. 4A and B). Importantly, the suppression of miR-486-5p for proliferation of NSCLC cells was impaired by GAB2 vector (Fig. 4C). Moreover, the inhibitory action of miR-486-5p for cell invasion almost disappeared in GAB2 overexpression group (Fig. 4D). All these findings indicated that miR-486-5p inhibited cell proliferation and invasion to a certain extent through the regulation of GAB2 in NSCLC.

\section{Discussion}

A full understanding of the occurrence and development mechanisms of NSCLC is necessary for opening a new pathway for NSCLC patients to improve survival. We conducted this research to explore the alteration of miR-486-5p expression and its effect on the progression of NSCLC. Moreover, miR-486-5p expression levels were declined in NSCLC which promoted cell proliferation and invasion in NSCLC. Additionally, GAB2 was confirmed as a direct target gene of miR-486-5p in NSCLC. We considered that miR-486-5p would repress NSCLC development by affecting GAB2 expression, indicating that miR-486-5p had a suppressive effect on NSCLC progression.

In various human cancers, miR-486 is usually expressed aberrantly and has inhibitory action. Previous studies demonstrated that miR-486 could be used as a biomarker for early diagnosis and recurrence of NSCLC (23), and had an inhibitory action for the growth and development of NSCLC (24). Other researchers also found the downregulation of miR-486 in prostatic carcinoma (25) and esophageal cancer (26) which was similar to our results. Additionally, low miR-486-5p expression was identified in NSCLC and promoted tumor metastasis and development by regulating ARHGAP5 (27). Our findings also suggested miR-486-5p repressed cell proliferation and invasion by suppressing GAB2 in NSCLC. Although miR-486 was confirmed to associate with tumorigenesis and progression of NSCLC, its relationship with GAB2 in NSCLC is still not clear.
Grb2-associated binding protein 2 (GAB2) was frequently detected in many human malignancies (28). As an oncogene, GAB2 has been identified in glioblastoma (29), gastric cancer (30) and renal cell carcinoma (31). Previously, GAB2 was identified to express highly in lung cancer (32). We found the upregulation of GAB2 in NSCLC as well. Functionally, GAB2 promoted cell proliferation and invasion in NSCLC. Similar to our results, GAB2 was also reported to have a positive impact on cell migration in NSCLC via Akt signaling pathway (33). Based on these results, we considered that GAB2 obviously promoted growth and metastasis of NSCLC. Hence, the low expression of GAB2 induced by miR-486-5p could help repress NSCLC development. However, due to the poor current laboratory conditions, there are still several limitations in this research such as the deficiency of in vivo experiments. We will further perform the experiments to validate our conclusion in future research.

In conclusion, we found that miR-486-5p was downregulated in NSCLC. Moreover, miR-486-5p inhibited cell proliferation and invasion through repressing GAB2 in NSCLC. This new pathway may help us better understand NSCLC pathogenesis and provide beneficial clues for the diagnosis and treatment of NSCLC.

\section{Acknowledgements}

Not applicable.

\section{Funding}

No funding was received.

\section{Availability of data and materials}

The datasets used and/or analyzed during the present study are available from the corresponding author on reasonable request.

\section{Authors' contributions}

SY contributed significantly to the study design and data acquisition; SG performed the data analyses and wrote the manuscript. YH contributed to the conception of the study. All authors read and approved the final study.

\section{Ethics approval and consent to participate}

The study was approved by the Ethics Committee of The Central Hospital of Wuhan, Tongji Medical College, Huazhong University of Science and Technology (Wuhan, China). Signed informed consents were obtained from the patients or the guardians.

\section{Patient consent for publication}

Not applicable.

\section{Competing interests}

The authors declare that they have no competing interests. 


\section{Reference}

1. Chen W, Zheng R, Baade PD, Zhang S, Zeng H, Bray F, Jemal A, Yu XQ and He J: Cancer statistics in China, 2015. CA Cancer J Clin 66: 115-132, 2016.

2. Torre LA, Bray F, Siegel RL, Ferlay J, Lortet-Tieulent J and Jemal A: Global cancer statistics, 2012. CA Cancer J Clin 65: 87-108, 2015.

3. Fassina A, Cappellesso R and Fassan M: Classification of non-small cell lung carcinoma in transthoracic needle specimens using microRNA expression profiling. Chest 140: 1305-1311, 2011 .

4. Garzon R, Calin GA and Croce CM: MicroRNAs in cancer. Annu Rev Med 60: 167-179, 2009.

5. Bartel DP: MicroRNAs: Target recognition and regulatory functions. Cell 136: 215-233, 2009.

6. Oom AL, Humphries BA and Yang C: MicroRNAs: Novel players in cancer diagnosis and therapies. BioMed Res Int 2014: 959461, 2014.

7. Li J, Wang Y, Luo J, Fu Z, Ying J, Yu Y and Yu W: miR-134 inhibits epithelial to mesenchymal transition by targeting FOXM1 in non-small cell lung cancer cells. FEBS Lett 586: 3761-3765, 2012

8. Wang P, Lv HY, Zhou DM and Zhang EN: miR-204 suppresses non-small-cell lung carcinoma (NSCLC) invasion and migration by targeting JAK2. Genet Mol Res 15: gmr6415, 2016.

9. Zhou YL, Xu YJ and Qiao CW: MiR-34c-3p suppresses the proliferation and invasion of non-small cell lung cancer (NSCLC) by inhibiting PAC1/MAPK pathway. Int J Clin Exp Pathol 8: 6312-6322, 2015.

10. Xiao P, Liu W and Zhou H: miR-200b inhibits migration and invasion in non-small cell lung cancer cells via targeting FSCN1. Mol Med Rep 14: 1835-1840, 2016.

11. Zhang JG, Wang JJ, Zhao F, Liu Q, Jiang $\mathrm{K}$ and Yang GH: MicroRNA-21 (miR-21) represses tumor suppressor PTEN and promotes growth and invasion in non-small cell lung cancer (NSCLC). Clin Chim Acta 411: 846-852, 2010.

12. Duan B, Guo T, Sun H, Cai R, Rui Q and Xi Z: miR-205 as a biological marker in non-small cell lung cancer. Biomed Pharmacother 91: 823-830, 2017.

13. Ye L, Wang H and Liu B: miR-211 promotes non-small cell lung cancer proliferation by targeting SRCIN1. Tumour Biol 37: $1151-1157,2016$

14. Li T, Ding ZL, Zheng YL and Wang W: MiR-484 promotes non-small-cell lung cancer (NSCLC) progression through inhibiting Apaf-1 associated with the suppression of apoptosis. Biomed Pharmacother 96: 153-164, 2017.

15. Sun H, Cui C, Xiao F, Wang H, Xu J, Shi X, Yang Y, Zhang Q, Zheng X, Yang X, et al: miR-486 regulates metastasis and chemosensitivity in hepatocellular carcinoma by targeting CLDN10 and CITRON. Hepatol Res 45: 1312-1322, 2015.

16. Tan K, Huang G and Fang Q: MiR-486-5p prevents migration, invasion and EMT by regulating smad 2 in breast cancer. Int $\mathrm{J}$ Clin Exp Med 10: 8942-8949, 2017.

17. Alexander MS, Casar JC, Motohashi N, Vieira NM, Eisenberg I, Marshall JL, Gasperini MJ, Lek A, Myers JA, Estrella EA, et al: MicroRNA-486-dependent modulation of DOCK3/PTEN/AKT signaling pathways improves muscular dystrophy-associated symptoms. J Clin Invest 124: 2651-2667, 2014

18. Xu J,Li R, Workeneh B, Dong Y, Wang X and Hu Z: Transcription factor FoxO1, the dominant mediator of muscle wasting in chronic kidney disease, is inhibited by microRNA-486. Kidney Int 82: 401-411, 2012.
19. Li Y, Ma C, Qian M, Wen Z, Jing H and Qian D: Downregulation of NOB1 suppresses the proliferation and tumor growth of non-small cell lung cancer in vitro and in vivo. Oncol Rep 31: 1271-1276, 2014.

20. Wang Y, Li Y, Wu B, Shi C and Li C: MicroRNA-661 promotes non-small cell lung cancer progression by directly targeting RUNX3. Mol Med Rep 16: 2113-2120, 2017.

21. Ke Y, Zhao W, Xiong J and Cao R: MiR-149 inhibits non-smallcell lung cancer cells emt by targeting FOXM1. Biochem Res Int 2013: 506731, 2013.

22. Livak KJ and Schmittgen TD: Analysis of relative gene expression data using real-time quantitative PCR and the 2(-Delta Delta C(T)) method. Methods 25: 402-408, 2001.

23. Li W, Wang Y, Zhang Q, Tang L, Liu X, Dai Y, Xiao L, Huang S, Chen L, Guo Z, et al: Correction: MicroRNA-486 as a biomarker for early diagnosis and recurrence of non-small cell lung cancer. PLoS One 11: e0148589, 2016.

24. Shao Y, Shen YQ, Li YL, Liang C, Zhang BJ, Lu SD, He YY, Wang P, Sun QL, Jin YX, et al: Direct repression of the oncogene CDK 4 by the tumor suppressor miR-486-5p in non-small cell lung cancer. Oncotarget 7: 34011-34021, 2016.

25. Zhang $X$, Zhang T, Yang K, Zhang M and Wang K: miR-486-5p suppresses prostate cancer metastasis by targeting Snail and regulating epithelial-mesenchymal transition. OncoTargets Ther 9: 6909-6914, 2016.

26. Yi Y, Lu X, Chen J, Jiao C, Zhong J, Song Z, Yu X and Lin B: Downregulated miR-486-5p acts as a tumor suppressor in esophageal squamous cell carcinoma. Exp Ther Med 12: 3411-3416, 2016.

27. Wang J, Tian X, Han R, Zhang X, Wang X, Shen H, Xue L, Liu Y, Yan X, Shen J, et al: Downregulation of miR-486-5p contributes to tumor progression and metastasis by targeting protumorigenic ARHGAP5 in lung cancer. Oncogene 33: 1181-1189, 2014.

28. Ding CB, Yu WN, Feng JH and Luo JM: Structure and function of Gab2 and its role in cancer (Review). Mol Med Rep 12: 4007-4014, 2015

29. Tian LQ, Liu EQ, Zhu XD, Wang XG, Li J and Xu GM: MicroRNA-197 inhibits cell proliferation by targeting GAB2 in glioblastoma. Mol Med Rep 13: 4279-4288, 2016.

30. Lee SH, Jeong EG, Nam SW, Lee JY, Yoo NJ and Lee SH: Increased expression of Gab2, a scaffolding adaptor of the tyrosine kinase signalling, in gastric carcinomas. Pathology 39: 326-329, 2007.

31. Gu DH, Mao JH, Pan XD, Zhu H, Chen X, Zheng B and Shan Y: microRNA-302c-3p inhibits renal cell carcinoma cell proliferation by targeting Grb2-associated binding 2 (Gab2). Oncotarget 8: 26334-26343, 2017.

32. Xu XL, Wang X, Chen ZL, Jin M, Yang W, Zhao GF and Li JW: Overexpression of Grb2-associated binder 2 in human lung cancer. Int J Biol Sci 7: 496-504, 2011.

33. Xu LJ, Wang YC, Lan HW, Li J and Xia T: Grb2-associated binder-2 gene promotes migration of non-small cell lung cancer cells via Akt signaling pathway. Am J Transl Res 8: 1208-1217, 2016.

This work is licensed under a Creative Common Attribution-NonCommercial-NoDerivatives 4.0 International (CC BY-NC-ND 4.0) License. 